\title{
Systems and technologies for objective evaluation of technical skills in laparoscopic surgery
}

\author{
JUAN A SÁNCHEZ-MARGALLO, FRANCISCO M SÁNCHEZ-MARGALLO , \\ IGNACIO OROPESA, ENRIQUE J GÓMEZ
}

\begin{abstract}
Minimally invasive surgery is a highly demanding surgical approach regarding technical requirements for the surgeon, who must be trained in order to perform a safe surgical intervention. Traditional surgical education in minimally invasive surgery is commonly based on subjective criteria to quantify and evaluate surgical abilities, which could be potentially unsafe for the patient. Authors, surgeons and associations are increasingly demanding the development of more objective assessment tools that can accredit surgeons as technically competent. This paper describes the state of the art in objective assessment methods of surgical skills. It gives an overview on assessment systems based on structured checklists and rating scales, surgical simulators, and instrument motion analysis. As a future work, an objective and automatic assessment method of surgical skills should be standardized as a means towards proficiency-based curricula for training in laparoscopic surgery and its certification.
\end{abstract}

Key words: Laparoscopic surgery, objective evaluation, motion analysis, surgical simulators, psychomotor skills

\section{Introduction}

Minimally invasive surgery is a highly demanding surgical approach regarding technical requirements for the surgeon such as the use of new surgical instruments, which are longer than the instruments used in open surgery, the lack of haptic feedback, the fulcrum effect, and the loss of depth perception. These aspects require new technical skills for the surgeon, which must be trained in order to perform a safe surgical intervention. Therefore, it is useful to know the psychomotor skills of surgeons during their training program, as an essential part of the assessment of their surgical proficiency.

Traditional surgical education in minimally invasive surgery is commonly based on subjective criteria. An experienced surgeon, who observes the evolution of the trainee, makes the evaluation of his/her surgical skills. Then, trainees are allowed to contribute to the surgical intervention under the supervision of an experienced surgeon. However, throughout this training methodology surgical skills cannot be precisely assessed and may be affected by bias. For this reason, authors, surgeons and associations are increasingly demanding the development of more objective assessment tools that can accredit surgeons as technically competent (1-3). Nevertheless, nowadays there is not a universally extended or recommended system to be used as an objective evaluation tool of technical skills $(1,4)$.

To perform the surgical training and evaluation, there is a tendency to move the early stages of training, concerning the acquisition of motor skills, outside the operating room. There has been an evolution in the development of physical, virtual and hybrid simulators for training in laparoscopic surgery, which are a suitable environment for learning surgical skills away from the clinical responsibility of putting a patient at risk.

Several objective assessment techniques have been proposed, most of them based on structured rating 
scales scored by experts (5-7). In general terms, these techniques perform the evaluation through rated checklists on an inanimate benchtop model. These tests normally consist of stations where a trainee performs a surgical task for a group of faculty observers. With video assessment methods the recorded surgical performance is subsequently rated by the assessor, thus providing more flexibility (8).

The development of virtual simulators increases the automation of the evaluation process based on the user interaction with a virtual surgical scenario and a pair of instruments with simulated haptic feedback $(9,10)$. The lack of realism is covered by physical $(11,12)$ and hybrid simulators $(13)$, which also implement automatic tools for the objective assessment of technical skills. Finally, another attempt to develop more automatic objective evaluation methods are the systems based on the motion analysis of surgical instruments (14-16). These systems compute metrics to quantify motion information regarding the use of the surgical instruments to establish the technical skill level of the surgeon.

This article reviews the different approaches developed to address the problem of objective evaluation of technical surgical skills, providing different options to be used in clinical practice and training curricula. It gives an overview of existing systems, how these systems work their advantages, and their drawbacks. The document has been organized in three main sections. In the first section, we will revise the most significant objective assessment methods of surgical skills based on structured rating scales. Subsequently, we will analyse the main types of surgical simulators for training and evaluation. Finally, we will focus our attention on motion analysis of laparoscopic instruments as a means to objectively assess surgical dexterity.

\section{Structured rating systems for objective evaluation}

\section{Rating scales}

Rating scales are basic assessment methods that can be applied to several disciplines, including evaluation of surgical skills. One of the most generic types is the Global Rating System (GRS) (7), which is a nonblinded evaluation method consisting of a number of items as general markers of technical skills that could be applied to a wide variety of procedures, although not to specific tasks. This system needs an instructed examiner during the evaluation procedure, which could be affected by bias due to the nonblinded process. Video assessment methods using GRS such as the Operative Component Rating Scale (OCRS) (17) provide a more objective blinded rating process with the same scoring criteria as live rating systems (8).

The Objective Structured Clinical Examination (OSCE) was described by Harden et al. in order to avoid many of the disadvantages associated with traditional examination of clinical competence. A typical OSCE consists of a series of stations through which trainees rotate. At each station the trainee is asked to perform a specific evaluation task, which is assessed by an experienced surgeon using checklists or global rating scales. However, there is no specific part focused on technical skills.

The Objective Structured Assessment of Technical Skills (OSATS) was developed following the success of the OSCE system in assessing surgical competences. This is a performance-based assessment of technical skills, in which trainees perform a series of timelimited surgical tasks at each station (5). It involves the use of two scoring systems: A task-specific checklist score and a global rating scale of overall performance. Although OSATS was first validated for use with a benchtop model, it can also be applied to physical laparoscopic simulators and in the operating theatre (18). A most specific version of the OSATS method is the Global Operative Assessment of Laparoscopic Skills (GOALS), which provides a method based on a global rating system for evaluation of operative performance during a laparoscopic procedure (6). This assessment tool rates depth perception, bimanual dexterity, efficiency, tissue handling, and autonomy.

\section{Scoring systems}

Nowadays, one of the most extended objective scoring systems for evaluation of surgical skills is the Fundamentals of Laparoscopic Surgery (FLS) developed by the Society of American Gastrointestinal Endoscopic Surgeons (SAGES) (19). This program consists of web-based study guides and hands-on manual skills practice and training. The manual skills assessment is based on the McGill Inanimate System for Training and Evaluation of Laparoscopic Skills (MISTELS), which consists of a series of five tasks with an objective scoring system (20). It uses time and accuracy (number of errors) in order to assess each individual task.

\section{Surgical simulators for training and evaluation}

In the last decade there has been an increment in the use of surgical simulators for training of surgical skills, mainly during the early phases of the training curricula, avoiding putting patients at risk. Simulators can be classified according to their degree of realism into virtual 

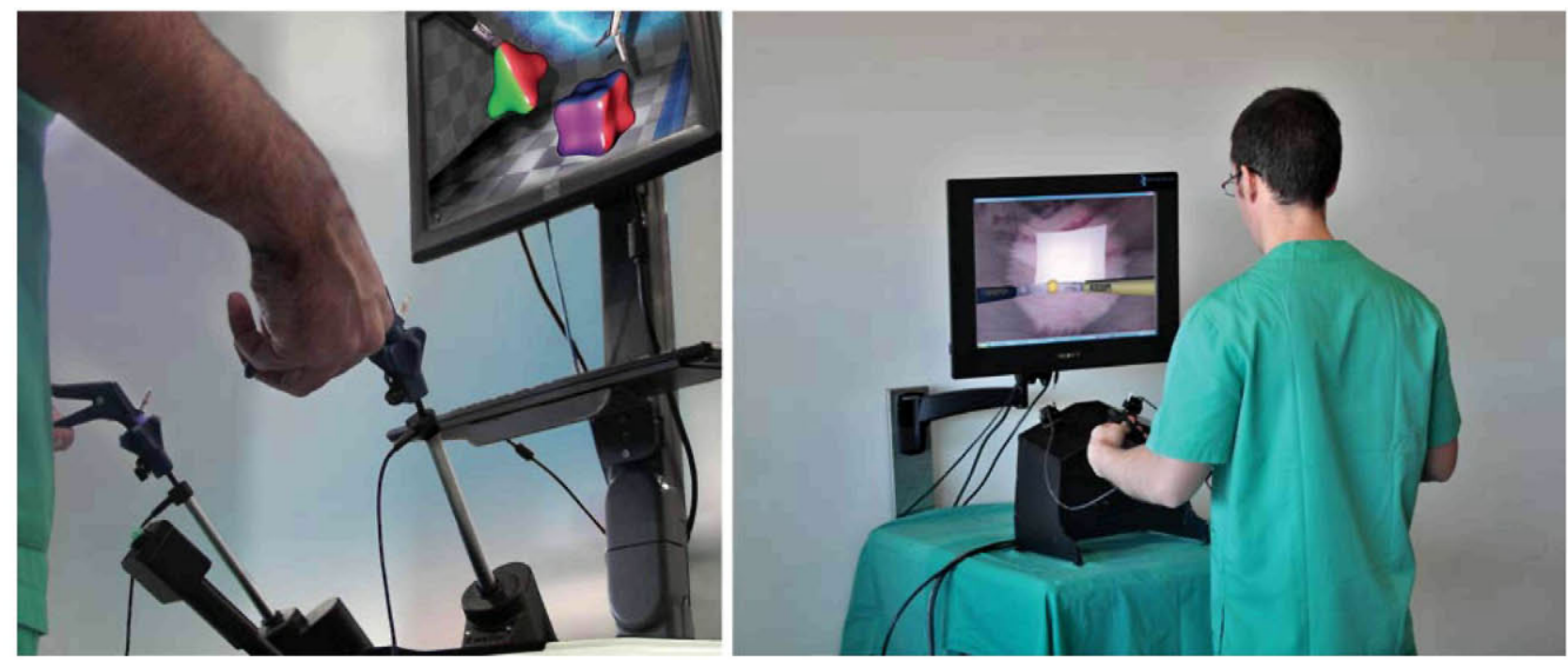

Figure 1. Left: LapMentor surgical simulator. Right: SINERGIA Virtual simulator.

(no real scenarios), physical (no virtual scenarios) or hybrid (combination of real and virtual scenarios).

\section{Virtual simulators}

The introduction of virtual simulators as a training and evaluation tool for laparoscopic surgery led to the replacement of real patients by computer images (Figure 1) (21-25). Virtual reality simulators provide controlled training and objective skills assessment on exercises ranging form simple tasks $(22,24)$ to complex laparoscopic procedures $(9,10,21,23,25)$. These devices are continuously improving the visual realism of their scenarios $(9,10,21)$ and force feedback $(9,21-23)$.
The current principal laparoscopic simulators and some of their main features can be found in Table I $(9,10,21-32)$. To characterize each of them we can establish

- the available kind of scenarios for training and surgical assessment,

- whether they offer force feedback, and

- their positive validation studies.

\section{Physical simulators}

Currently there are several physical laparoscopic simulators, which provide additional tools to perform an objective evaluation of surgeons' technical skills (Figure 2, Table II). The Computer Enhanced

Table I. Virtual reality simulators. Characteristics and validation.

\begin{tabular}{|c|c|c|c|}
\hline Device & Force feedback & Training/evaluation scenarios & Validation \\
\hline LapMentor (Simbionix Ltd., Beit Golan, Israel) & Yes & $\begin{array}{l}\text { Simple and advanced tasks } \\
\text { Procedures }\end{array}$ & $\begin{array}{l}\text { Construct (21) } \\
\text { Concurrent (26) } \\
\text { Predictive (27) }\end{array}$ \\
\hline SINERGIA (SINERGIA Consortium) & Supported & Simple tasks & Construct (22) \\
\hline LapSim (Surgical Science, Gothenburg, Sweden) & Supported & Simple and advanced tasks & $\begin{array}{l}\text { Construct (23) } \\
\text { Concurrent (28) } \\
\text { Predictive (29) }\end{array}$ \\
\hline MIST-VR (Mentice AB, Göteborg, Sweden) & No & Simple tasks & $\begin{array}{l}\text { Construct (24) } \\
\text { Concurrent (30) } \\
\text { Predictive (31) }\end{array}$ \\
\hline SIMENDO (DeltaTech, Delft, Netherlands) & No & Simple and advanced tasks & $\begin{array}{l}\text { Construct (25) } \\
\text { Concurrent (32) }\end{array}$ \\
\hline SEP Simulator (SimSurgery AS, Oslo, Norway) & No & $\begin{array}{l}\text { Simple and advanced tasks } \\
\text { Procedures }\end{array}$ & Construct (10) \\
\hline LapVR (CAE Helthcare, Mainz, Germany) & Yes & $\begin{array}{l}\text { Simple and advanced tasks } \\
\text { Procedures }\end{array}$ & Construct (9) \\
\hline
\end{tabular}




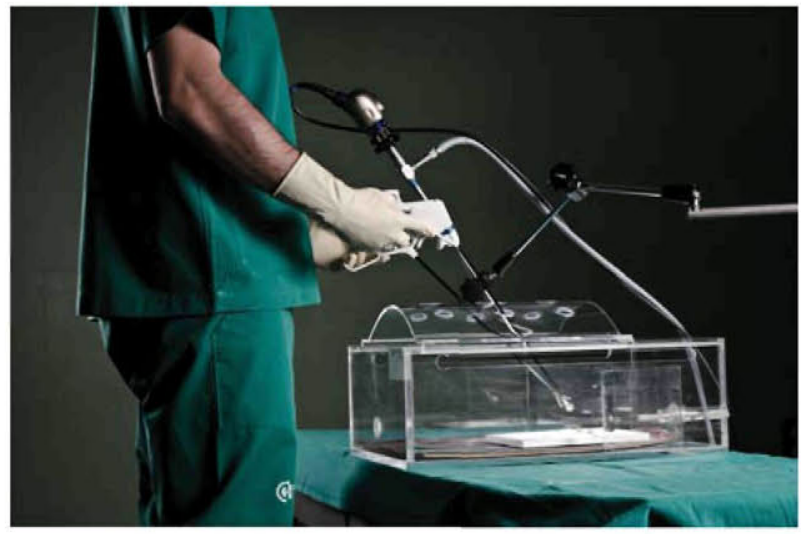

Figure 2. Basic physical simulator for laparoscopic training.

Laparoscopic Training System (CELTS) (24) is a computer-based laparoscopic trainer capable of tracking the motion of two laparoscopic instruments through a modified Virtual Laparoscopic Interface (VLI) (Immersion Corp., San Jose, CA, USA). It evaluates a set of objective metrics based on instrument motion.

A combination of ultrasound technology and physical simulator is another approach to objectively assess the surgical performance. Sokollik et al. (33) define a system that determines the position and rotation of the instruments using miniature ultrasound transmitters (Zebris Medical GmbH, Isny, Germany) placed on them. It provides two training modules: An aiming task with seven LED targets to be touched and a suturing task. The ultrasound transmitter can be sterilized, making it viable for use in the operating room.

The SurgicalSIM LTS simulator (Blackdust Design, Los Angeles, CA, USA) (12) is a system with sensors embedded within physical modules arranged for assessing the performance of validated exercises such as cannulation, cutting and suturing skills, where it is able to verify the knot integrity.

The Hiroshima University Endoscopy Surgical Assessment Device (HUESAD) was designed to evaluate the smoothness in the use of laparoscopic instruments $(11,34,35)$. It consists of an accurate tracking device for real laparoscopic instruments, an experimental table, a monitor, and a personal computer. It provides simple evaluation tasks for assessment of visuo-spatial skills such as touching a set of poles placed in different locations of the experimental table.

Other approaches combine mechanical technology with a physical simulator. This is the case of the Advanced Dundee Endoscopic Psychomotor Tester (ADEPT) (36), which uses a set of potentiometers mounted in a dual-gimbal mechanism for tracking two real instruments. This system computes the instrument error, execution time, and task completion as evaluation parameters.

Table II summarizes some of the main characteristics of previous systems. It presents

- the kind of technology used,

- the objective evaluation criteria,

- whether the system is portable, and

- its validity as an assessment tool of surgical skills.

\section{Hybrid simulators}

These systems use computer vision techniques to combine a virtual setting with real training performance, creating augmented reality scenarios for surgical training and evaluation. One of the most extended is the ProMIS augmented reality simulator (CAE Helthcare, Mainz, Germany) (13). This system uses motion analysis techniques to track artificial markers on the instrument, using a stereoscopic camera system placed inside the simulator. It implements augmented reality techniques to insert visual information inside the surgical scenario so as to support the surgical procedure. This system provides objective evaluation based on the execution time and motion parameters such as path length of the instruments and motion smoothness. Another hybrid system (37), with similar technical characteristics to ProMIS, provides a dynamic artificial environment in order to insert visual support content and re-display training performances.

\section{Technologies for instrument motion analysis}

Motion analysis has been demonstrated to be an effective assessment tool for laparoscopic psychomotor

Table II. Physical simulators with tools for objective assessment of technical skills. Summary of some characteristics.

\begin{tabular}{lllll}
\hline Device & \multicolumn{1}{c}{ Technology } & Objective evaluation & Portable & Validation \\
\hline HUESAD (34) & Optical & Instrument motion & No & Construct (11,35) \\
Zebris (33) & Acoustic & Instrument motion & Yes & - \\
SurgicalSIM LTS & Sensor-based & Physical models with sensors & Yes & Construct (12) \\
CELTS & Optical & Instrument motion & Yes & Construct (24) \\
ADEPT & Gimbal mechanism & Instrument motion & - & Construct (36) \\
\hline
\end{tabular}


skills $(14,15)$. In general terms, a tracking system is composed of an object to be tracked, a system to track the target, and a system to process the positional information. If we organized tracking systems regarding the scenario in which they track the laparoscopic instruments, inside the patient or simulator and outside, we have intra-corporeal and extra-corporeal tracking systems.

\section{Extra-corporeal systems}

These systems are based on artificial visual markers (active and passive), sensors or mechanisms placed on the external part of the instrument. Mechanical systems estimate the position and orientation of the laparoscopic instruments based on encoders and potentiometers such as the Red Dragon (38), and its previous version the Blue Dragon, which also measure the torque and force applied by the surgeon.

Electromagnetic technology is a very extended technology concerning tracking of surgical instruments. Some studies use this technology for surgical skills assessment during a suturing task (39). On the other hand, the Imperial College Surgical Assessment Device (ICSAD) (40) uses an electromagnetic sensor attached to the dorsum of each hand in order to analyse the surgeon's performance.

Motion analysis systems based on infrared (IR) technology can be classified as active or passive systems. Active systems attach IR markers to the target and two or three cameras with an IR filter track them (41). Since the IR markers must be powered, they are wired. Passive tracking systems use retroreflective markers, which are illuminated by the camera in the IR spectrum. In this case markers are not wired. Some tracking systems such as Polaris ${ }^{\circledR}$ (NDI; Waterloo, Canada) can be used as active or passive systems (42). Hwang et al. (43) developed a hybrid approach with a passive optical system and an electromagnetic tracking system in order to minimize the effects of optical occlusions and electromagnetic distortions.

Extra-corporeal video-based tracking systems work in the visible spectrum and use a set of landmarks placed on the instruments to be tracked by a camera system. Landmarks are usually planar patterns with geometrical restrictions and binary colour to provide maximum contrast in the images. One example of this tracking technology for motion analysis of surgical instruments is the MicronTracker ${ }^{\circledR}$ (Claron Technology Inc., Toronto, Canada) (44).

Concerning sensor-based tracking of surgical instruments, the Delft University of Technology (Delft, the Netherlands) developed a device consisting of a two-gimbal mechanism with three optical computer mouse sensors (14). On the other hand, other approaches tend to use miniature inertial sensors combined with electromagnetic technology (45).

\section{Intra-corporeal systems}

These approaches track the position of the laparoscopic instruments from inside the human body or surgical simulator. In this case, the use of electromagnetic technology for assessment of technical skills based on instrument motion requires very smallsize sensors that are attached on the distal part of the instruments. Some researchers attach the electromagnetic sensors on the instrument's shaft and near the tip (16), while others embed them into the instrument's shaft (46).

The use of video from the laparoscope or the camera inside the simulator as a source of information to perform the instrument tracking is another possible approach. Some of these systems use artificial markers on the instruments such as colour markers on the distal end of the instrument. These markers are tracked using computer vision techniques including colour segmentation (47), stereoscopic techniques (37), or a Continuously Adaptive Mean Shift (CAMSift) algorithm (48). Tonet et al. (49) compute the third dimensional position of the instrument tip according to the angle between the edges of the colour marker, previously identified by the Hough Transform. Another possibility is to use a set of tapes placed near the instrument tip as a reference point and then to compute the depth of the instrument by means of analysing the variation of the marker's diameter, as described by Zhang et al. (50). They use three tapes in case one or two of them were occluded during the surgical intervention. Another approach based on landmarks (51) uses a cover for the instrument with a light pattern made of LEDs.

The use of landmarks inside the patient is always a critical issue concerning their biocompatibility. In order to avoid them, some researchers analyse the $3 \mathrm{D}$ position of the instrument tip based on its projection in the image plane (Figure 3). Additionally, Voros et al. (52) restrict the search of the laparoscopic instrument by means of computing the projection of the instrument's insertion point into the abdominal cavity. Other approaches use a Bayesian classifier (53) or a Cascade of Boosted Classifiers (54) previously trained to identify the laparoscopic instrument (Figure 4). In order to track the identified instrument over time one of the most extended methods is the use of particle filters (55).

A summary of the reviewed technologies for laparoscopic instruments tracking is presented in Table III. 

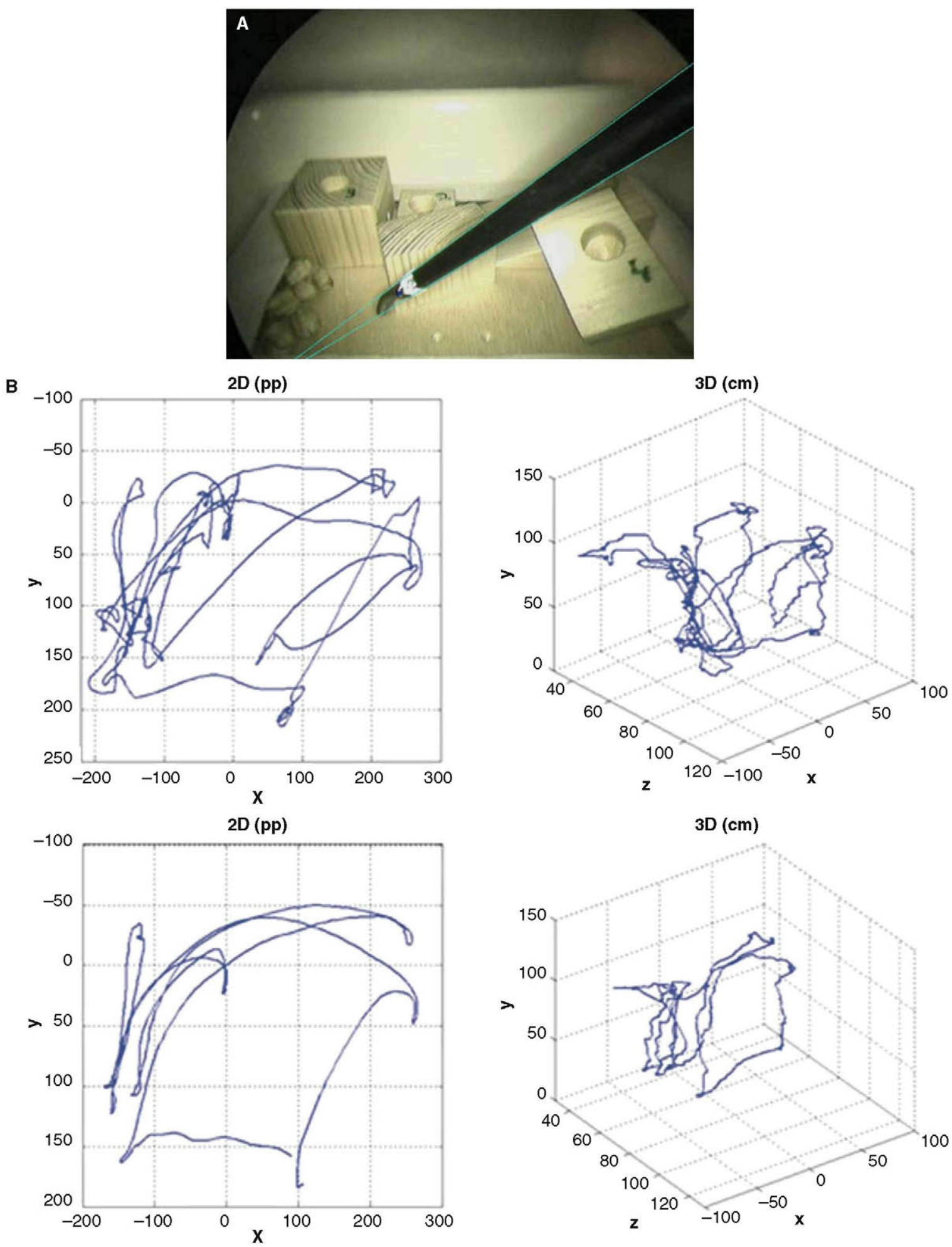

Figure 3. (A) Laparoscopic instrument tracking based on endoscopic video analysis for psychomotor skills assessment (EVA system) (15). (B) Tip motion representation examples, the top graphs are from a novice surgeon, and the bottom from an expert.

Table IV shows a review of the different motion metrics and the studies that have applied them for objective evaluation of psychomotor skills in minimally invasive surgery.

\section{Discussion}

Evolution from traditional to laparoscopic surgery has made it necessary for surgeons to learn new 

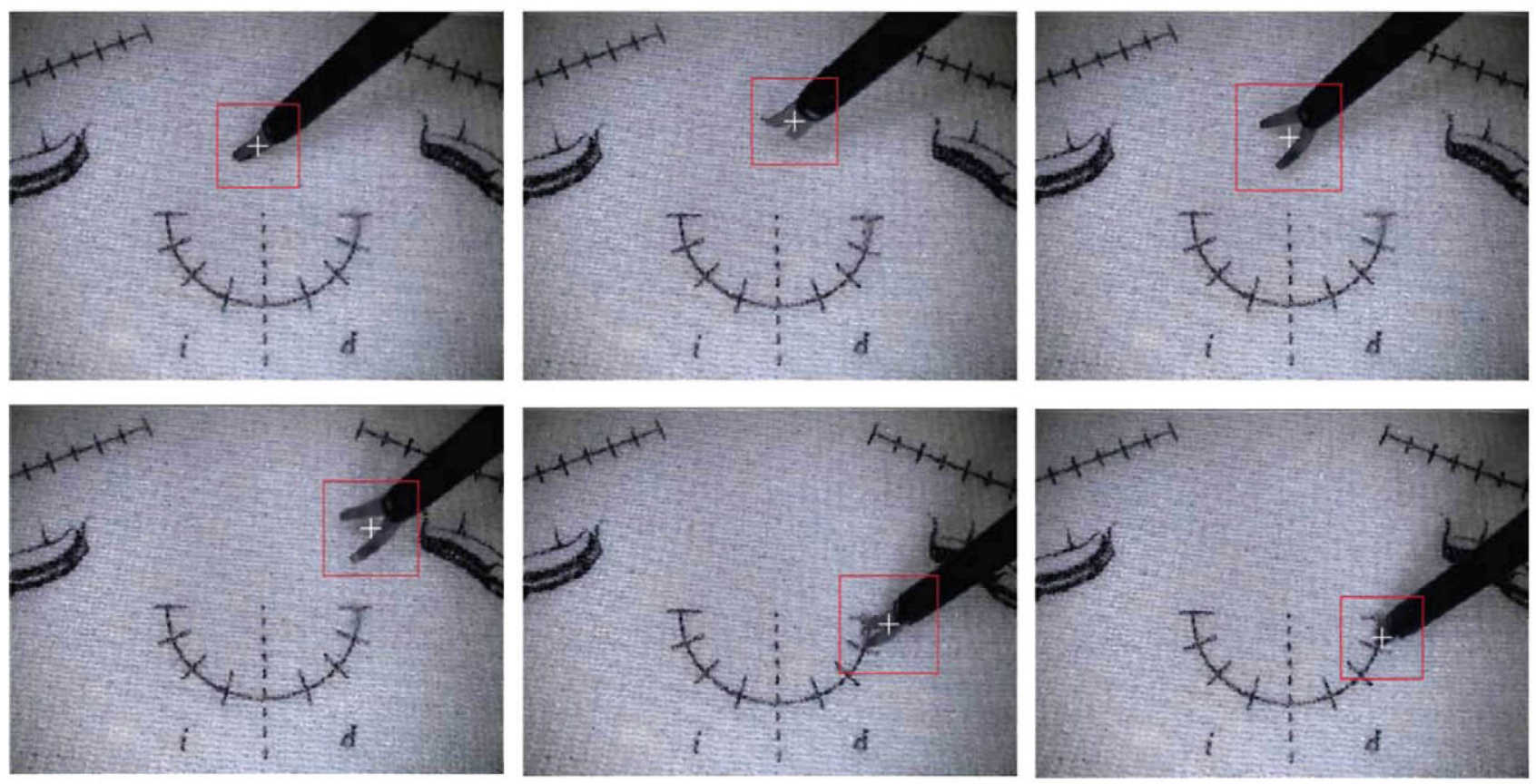

Figure 4. Example of an automatic tracking method of the tip of the laparoscopic scissors based on an Adaptive Boosting learning algorithm (54). This sequence of images corresponds to a basic laparoscopic training task of cutting.

psychomotor skills, which must be trained, evaluated, and certified. In this article, existent methods and tools for the objective assessment of laparoscopic surgical skills have been presented. These systems attempt to replace the traditional methods based on subjective criteria, incorporating a whole new range of objective metrics to quantify surgical performance.
Most of the assessment methods based on structured checklists and rating scales $(5,7)$ are valid for feedback or measuring progress of training, but few can be used for examination or credentialing (1). These assessment systems are easy to implement within a training program because of their simplicity, although they are time-consuming procedures and

Table III. Technologies applied for laparoscopic instrument tracking. Summary of the main advantages and drawbacks.

\begin{tabular}{|c|c|c|}
\hline Technique & Advantages & Drawbacks \\
\hline Mechanical & $\begin{array}{l}\text { Excellent accuracy } \\
\text { Interference immunity } \\
\text { They can be sterilized for a real } \\
\text { surgical intervention }\end{array}$ & $\begin{array}{l}\text { Cumbersome handling } \\
\text { Limited range of movements due to physical connection } \\
\text { Not portable }\end{array}$ \\
\hline IR-based & $\begin{array}{l}\text { Reasonable range, accuracy, } \\
\text { and resolution }\end{array}$ & $\begin{array}{l}\text { Line-of-sight problems } \\
\text { Commercial devices are expensive }\end{array}$ \\
\hline Acoustic & Large range and low cost & $\begin{array}{l}\text { Slow update rates } \\
\text { Speed of sound affected by environmental conditions }\end{array}$ \\
\hline Electromagnetic & No line-sight problems & $\begin{array}{l}\text { Low latency } \\
\text { Affected by distortions from magnetic objects } \\
\text { Rapid decrease in accuracy/resolution with distance } \\
\text { Sensors on the instrument are wired }\end{array}$ \\
\hline Sensor-based & High update rates & $\begin{array}{l}\text { Accumulative error } \\
\text { They require wires to provide power }\end{array}$ \\
\hline Extra-corporeal video-based & $\begin{array}{l}\text { High accuracy } \\
\text { No magnetic distortions } \\
\text { Low cost }\end{array}$ & $\begin{array}{l}\text { Low accuracy } \\
\text { Line-of-sight problems } \\
\text { It is necessary to have landmarks on the instruments }\end{array}$ \\
\hline Intra-corporeal video-based & $\begin{array}{l}\text { No magnetic distortions } \\
\text { Low cost } \\
\text { Sometimes it is not necessary } \\
\text { to modify the surgical tools }\end{array}$ & $\begin{array}{l}\text { Low accuracy } \\
\text { They lose the instrument position when it is out } \\
\text { of the laparoscope's field of view }\end{array}$ \\
\hline
\end{tabular}


Table IV. Definition of the main assessment metrics based on instrument motion analysis and systems that use them for objective evaluation of surgical dexterity.

\begin{tabular}{|c|c|}
\hline Metric & System \\
\hline Time. Total time taken to perform the task (in s) & $(13-16,21-25,33,35,39,46)$ \\
\hline Approaching time. Time taken to reach the target point (in s) & (34) \\
\hline $\begin{array}{l}\text { Path length. Length of the curve described by the tip } \\
\text { of the instrument while performing the task (in } \mathrm{mm} \text { ) }\end{array}$ & $(13-16,21-25,33,39,46)$ \\
\hline $\begin{array}{l}\text { Distance efficiency. Relationship between measure path length and shortest } \\
\text { path to describe the economy of movements }(-)\end{array}$ & (33) \\
\hline Depth perception. It is the total distance travelled by the instrument along its axis (in $\mathrm{mm}$ ) & $(15,24)$ \\
\hline Transit profile. Transit trajectory projected onto plane $2 \mathrm{D}(-)$ & (33) \\
\hline Deviation on horizontal/vertical plane. Deviations from the ideal courses in these two directions (in $\mathrm{mm} \cdot \mathrm{s}$ ) & (35) \\
\hline Response orientation. It characterizes the amount of rotation about the axis of the instrument (in radians) & (24) \\
\hline Speed. Rate of change of the instrument's position (in $\mathrm{mm} / \mathrm{s}$ ) & $(15,16,33,39,43)$ \\
\hline Maximum speed value (in $\mathrm{mm} / \mathrm{s}$ ) & (11) \\
\hline Speed profile. Shape of the speed curve (-) & (33) \\
\hline Acceleration. Rate of change of the instrument's speed (in $\mathrm{mm} / \mathrm{s}^{2}$ ) & $(15,43)$ \\
\hline $\begin{array}{l}\text { Motion smoothness. A motion analysis parameter based on the third time-derivative of position, } \\
\text { which represents a change in acceleration (in } \mathrm{mm} / \mathrm{s}^{3} \text { ) }\end{array}$ & $(14,15,22,24,28,43,46)$ \\
\hline $\begin{array}{l}\text { Economy of area. Relationship between the maximum area occupied by } \\
\text { the instrument and the total path length }(-)\end{array}$ & $(15)$ \\
\hline $\begin{array}{l}\text { Economy of volume. Relationship between the maximum volume occupied } \\
\text { by the instrument and the total path length }(-)\end{array}$ & $(15)$ \\
\hline Search time. Percentage of time spent in the "search zone" (in \%) & (15) \\
\hline Idle time. Percentage of time where the instrument is considered to be still (in \%) & (15) \\
\hline $\begin{array}{l}\text { Number of movements. Number of movements made to complete the task } \\
\text { (zero crossings on the acceleration/time profile) }\end{array}$ & $(21,22,39)$ \\
\hline
\end{tabular}

they require one or several assessors present throughout the evaluation process. In addition, the subjectivity issues of assessors could lead to possible ambiguity in their scores. Video offline evaluation (8) provides more flexibility and help with feedback of the trained. However, it entails a high workload, as experts must analyse one by one all recorded videos for each task. Often only the laparoscopic camera view may blind the reviewer to certain aspects of the operation that could also be relevant for the evaluation.

The OSCE examination method is focused on the assessment of procedural knowledge and attitude of the trainee towards the patient, but it has no specific evaluation of psychomotor skills, which is included in the patient case domain. OSATS was one of the first methods designed for objective technical skills assessment and one of the few used in clinical practice (18), although with a low level of evidence. Implementation of the OSATS in the OR may present ambiguities in the scoring system, which could be influenced by many factors during the course of the intervention.

The training and accreditation program FLS only takes into account parameters such as completion time and procedural performance (accuracy error) for evaluation purposes $(19,20)$. However, to better understand surgical gestures and to exploit the possibilities that can offer tracking technologies, a wider range of metrics should be considered and analysed (see Table IV).

The use of surgical simulators provides trainees an environment always available for training and without the need of a supervisor, which reduces the associated costs. Although practice and assessment on a simulator are no substitute for surgical practise, they enable trainees to become competent in fundamental skills before entering the complex real surgical environment. Virtual simulators are ideal for monitoring the surgeon's learning curve, and offer a wide range of metrics that can be used for objective assessment $(9,10,21-25)$. However, some limitations have slowed down their clinical implantation, such as the cost resulting from the expensive technologies behind the simulators, which makes them unaffordable for some institutions and hospitals, the limitations in visual realism and interaction (haptic feedback), and the mentally-driven constraints of thinking of these devices as a videogame with no didactic value (56). Despite their limitations, studies have proved 
that some virtual simulators are capable of providing formative assessment $(1,56)$. They allow the acquisition of both efficiency (motion, force) and quality metrics thanks to the combination of computergenerated environments with tracking technologies. In addition, they enhance the trainee's training process providing immediate feedback on peformance.

A major advantage of physical and hybrid simulators is that they provide natural haptic feedback, and therefore a natural instrument-tissue interaction. In general, these systems consist of a physical device in which to perform the training tasks, and an additional evaluation system to record metrics and evaluate surgical performance. Most of the analysed physical simulators use motion metrics as a means of technical evaluation $(24,33,34,36)$, whereas the Surgical SIM LTS uses error scores (12). The possibility of inserting virtual support content during the training procedure by means of cutting-edge technologies such as augmented reality $(13,37,57)$ gives hybrid simulators a significant added value. Surgeons consider hybrid simulators more realistic, with high didactic value, better haptic feedback, and usefulness than virtual reality simulators (57). In contrast to virtual simulators, which have predefined training programs, physical and hybrid simulators are able to use a set of tasks from established training programs or develop their own set of tasks.

In order to investigate surgeons' skills of operation, it is reasonable to observe the behaviour of surgical instruments carefully. Different technologies have been used for tracking of laparoscopic instruments such as mechanical, IR-based, electromagnetic, acoustic, sensor-based or video-based. Motion analysis systems are cheaper alternatives to virtual simulators, enabling natural haptic feedback. They can be used in almost every setting, from physical simulators to the OR, but quality metrics are more complicated to obtain in these systems. Moreover, it is difficult to establish a minimum precision and accuracy needed for tracking systems, which in most cases depends on the surgical application $(25,41)$.

In general, extra-corporeal tracking systems are very accurate approaches. However, they use markers or sensors placed on the external part of the surgical instrument, normally on the handle, which may disturb the natural use of the instrument. One of the main problems of external instrument tracking systems is that small movements on the top of the laparoscopic instruments result in large movements of its tip. Despite their high positional accuracy, both IR-based systems active and passive depend on having a clear line of sight between the tracked tool and the camera system (15). Moreover, external video-based tracking systems are sensitive to some environmental conditions such as illumination and velocity and the orientation of the tracked tool (44).

Electromagnetic tracking systems have no problems with occlusions $(39,58)$, although the accuracy of position and orientation measurements with this technology could be degraded with magnetic field distortions caused by the metallic objects in the clinical scenario $(15,41)$. For intra-corporeal applications of this tracking technology, a drastic reduction of the sensor size, owing to the reduced workspace, is required (46). Regarding the use of mechanical trackers, they have to be attached to the surgical instruments, which limits their full freedom of movements (41). In most cases, due to the nature of the technology and materials used, they can be sterilized, and therefore be used inside the OR. Nevertheless, their usual configuration is bulky, and thus not easily portable. Finally, since ultrasound transmitters can be sterilized, they could be used inside a clinical setting (33). However, ultrasound transmission can be affected by the environmental conditions (temperature, humidity), occlusions, or the loss of energy of the ultrasound signal with distance.

In general, the use of sensor-based systems for instrument motion analysis solves the problems with occlusion and interference (14), but their implementation into the OR is a challenging process. Hybrid approaches for instrument motion analysis could be a suitable option in order to address limitations (occlusions, interference) of some of the tracking technologies analysed above $(43,45)$.

The non-invasive characteristics of tracking systems based on endoscopic image makes them fit for any training and evaluation scenario outside and inside the OR. They offer an affordable and portable assessment tool, which does not require additional equipment that could interfere with the surgical performance and natural use of the instrument. Nevertheless, in some cases natural visual features of the instruments are not discriminative enough to accurately distinguish the instrument in the surgical scenario. Some approaches address this problem by using artificial markers on the instrument $(48,49)$. However, some of these approaches have some complications including the ambiguous image structures (52) and occlusions by blood, organs or other instruments, or smoke caused by electrodissection $(37,47,49,54)$. Both real-time image processing and the third dimension of the instrument's position are critical factors in these instrument tracking methods.

Although accreditation at each surgical training level should be carried out before moving on to the next level, there is a lack of official consensus on the tasks, metrics and assessment methods to use $(1,3,4)$. Based on the literature and our own experience, we 
believe that virtual simulators and tracking technologies in physical simulators are most suitable for the assessment of dexterity during the first phases of training $(15,39,54,56)$. Besides, we think of endoscopic video-based trackers for laparoscopic instruments as a possible solution to interferences in the surgical flow caused by extra-corporeal tracking systems $(15,54)$.

In order to develop an advanced and comprehensive objective assessment, a three-dimensional evaluation of trainees is necessary to obtain the right balance of attributes: A good knowledge base, surgical judgement and technical skills (59). Traditional evaluation methods for minimally invasive surgery based on subjective criteria suggest that an objective and automatic assessment method of surgical skills should be standardized to cope with mentors' and trainees' tight schedules, saving when possible the extra cost of having an experienced surgeon during the entire evaluation process, and as a means towards proficiencybased curricula for training in laparoscopic surgery and its certification. In addition, the implementation of automatic objective evaluation methods of surgical skills would be a valuable tool for the detection of weaknesses and errors during task performance.

\section{Acknowledgements}

This study was supported in part by the Plan Regional de Investigación, Consejería de Empleo, Empresa e Innovación, Gobierno de Extremadura and the European Social Found (PRE08057).

Declaration of interest: The authors report no conflicts of interest. The authors alone are responsible for the content and writing of the paper.

\section{References}

1. van Hove PD, Tuijthof GJM, Verdaasdonk EG, Stassen LPS, Dankelman J. Objective assessment of technical surgical skills. Br J Surg. 2010;97:972-87.

2. Roberts KE, Bell RL, Duffy AJ. Evolution of surgical skills training. World J Gastroenterol. 2006;12:3219-24.

3. Ritchie WP. Basic certification in surgery by the American Board of Surgery (ABS). What does it mean? Does it have value? Is it relevant? A personal opinion. Ann Surg. 2004;239:133-9.

4. Darzi A, Smith S, Tafinder N. Assessing operative skill. Needs to become more objective. BMJ. 1999;318:887-8.

5. Chipman JG, Schmitz CC. Using objective structured assessment of technical skills to evaluate a basic skills simulation curriculum for first-year surgical residents. J Am Surg. 2009; 209:364-70.e2.

6. Gumbs AA, Hogle NJ, Fowler DL. Evaluation of resident laparoscopic performance using global operative assessment of laparoscopic skills. J Am Coll Surg. 2007;204:308-13.
7. Doyle JD, Webber EM, Sidhu RS. A universal global rating scale for the evaluation of technical skills in the operating room. Am J Surg. 2007;193:551-5.

8. Driscoll PJ, Paisley AM, Paterson-Brown S. Video assessment of basic surgical trainees' operative skills. Am J Surg. 2008; 196:265-72.

9. Iwata N, Fujiwara M, Kodera Y, Tanaka C, Ohashi N, Nakayama $G$, et al. Construct validity of the LapVR virtualreality surgical simulator. Surg Endosc. 2011;25:423-8.

10. Hessel M, Buzink SN, Schoot BC, Jakimowicz JJ. Face and Construct Validity of the SimSurgery SEP VR Simulator for Salpingectomy in Case of Ectopic Pregnancy. J Gynecol Surg. 2012; Epub ahead of print.

11. Egi $H$, Okajima $M$, Kawahara $T$, Yoshimitsu $M$, Sumitani $D$, Tokunaga $M$, et al. Scientific assessment of endoscopic surgical skills. Minim Invasive Ther Allied Technol. 2010; 19:30-4.

12. Mathis $\mathrm{KL}$, Wiegmann DA. Construct validation of a laparoscopic surgical simulator. Simul Healthc. 2007;2:178-82.

13. Leblanc F, Senagore AJ, Ellis CN, Champagne BJ, Augestad KM, Neary PC, et al. Hand-assisted laparoscopic sigmoid colectomy skills acquisition: augmented reality simulator versus human cadaver training models. J Surg Educ. $2010 ; 67: 200-4$.

14. Chmarra MK, Klein S, Winter JCF, Jansen FW, Dankelman J. Objective classification of residents based on their psychomotor laparoscopic skills. Surg Endosc. 2010;24: 1031-9.

15. Oropesa I, Sánchez-González P, Chmarra MK, Lamata P, Fernández A, Sánchez-Margallo JA, et al. EVA: laparoscopic instrument tracking based on endoscopic video analysis for psychomotor skills assessment. Surg Endosc. 2013;27:1029-39.

16. Yamaguchi $S$, Yoshida $D$, Kenmotsu $H$, Yasunaga $T$, Konishi K, Ieiri S, et al. Objective assessment of laparoscopic suturing skills using a motion-tracking system. Surg Endosc. 2011;25:771-5.

17. Dath D, Regehr G, Birch D, Schlachta C, Poulin E, Mamazza J, et al. Toward reliable operative assessment: the reliability and feasibility of videotaped assessment of laparoscopic technical skills. Surg Endosc. 2004;18:1800-4.

18. Larsen CR, Grantcharov $\mathrm{T}$, Schouenborg L, Ottosen C, Soerensen JL, Ottesen B. Objective assessment of surgical competence in gynaecological laparoscopy: development and validation of a procedure-specific rating scale. BJOG. 2008; 115:908-16.

19. Sroka G, Feldman LS, Vassiliou MC, Kaneva PA, Fayez R, Fried G. Fundamentals of Laparoscopic Surgery simulator training to proficiency improves laparoscopic performance in the operating room - a randomized controlled trial. Am J Surg. 2010;199:115-20.

20. Vassiliou MC, Ghitulescu GA, Feldman LS, Stanbridge D, Leffondré K, Sigman HH, et al. The MISTELS program to measure technical skill in laparoscopic surgery: evidence for reliability. Surg Endosc. 2006;20:744-7.

21. Aggarwal R, Crochet P, Dias A, Misra A, Ziprin P, Darzi A. Development of a virtual reality training curriculum for laparoscopic cholecystectomy. Br J Surg. 2009;96:1086-93.

22. Sánchez-Peralta LF, Sánchez-Margallo FM, MoyanoCuevas JL, Pagador JB, Enciso-Sanz S, SánchezGonzález P, et al. Construct and face validity of SINERGIA laparoscopic virtual reality simulator. Int J Comput Assist Radiol Surg. 2010;5:307-15.

23. Schreuder HW, van Dongen KW, Roeleveld SJ, Schijven MP, Broeders IA. Face and construct validity of virtual reality simulation of laparoscopic gynecologic surgery. Am J Obstet Gynecol. 2009;200:540.e1-8. 
24. Maithel S, Sierra R, Korndorffer J, Neumann P, Dawson S, Callery $M$, et al. Construct and face validity of MIST-VR, Endotower, and CELTS: are we ready for skills assessment using simulators? Surg Endosc. 2006;20:104-12.

25. Verdaasdonk EG, Stassen LP, Schijven MP, Dankelman J. Construct validity and assessment of the learning curve for the SIMENDO endoscopic simulator. Surg Endosc. 2007;21:1406-12.

26. Okrainec A, Tekian A, Aarts MA, Grantcharov T, Escallon J, Reznick R. Virtual reality training on basic laparoscopic tasks versus virtual reality training of an entire surgical procedure: A randomized controlled trial using real world operations as an outcome. Proceedings of Annual Meeting of the Association for Surgical Education, Toronto, Canada, 2008.

27. Greco E, Escallon J, Grantcharov T, Okrainec A. Do performance measures on the LapMentor VR simulator predict FLS performance? Can J Surg. 2008;51:15.

28. Newmark J, Dandolu V, Milner R, Grewal H, Harbison S, Hernandez E. Correlating virtual reality and box trainer tasks in the assessment of laparoscopic surgical skills. Am J Obstet Gynecol. 2007;197:546.e1-4.

29. Hassan I, Osei-Agymang T, Radu D, Gerdes B, Rothmund M, Fernández ED. Simulation of laparoscopic surgery-Four years' experience at the Department of Surgery of the University Hospital Marburg. Wien Klin Wochenschr. $2008 ; 120: 70$.

30. Gallagher AG, Lederman AB, McGlade $\mathrm{K}$, Satava RM, Smith CD. Discriminative validity of the Minimally Invasive Surgical Trainer in Virtual Reality (MIST-VR) using criteria levels based on expert performance. Surg Endosc. 2004;18: $660-5$.

31. Stefanidis D, Korndorffer JR, Black FW, Dunne JB, Sierra R, Touchard CL, et al. Psychomotor testing predicts rate of skill acquisition for proficiency-based laparoscopic skills training. Surgery. 2006;140:252-62.

32. Verdaasdonk EG, Stassen LP, Monteny LJ, Dankelman J. Validation of a new basic virtual reality simulator for training of basic endoscopic skills: the SIMENDO. Surg Endosc. 2006;20:511-18.

33. Sokollik C, Gross J, Buess G. New model for skills assessment and training progress in minimally invasive surgery. Surg Endosc. 2004; 18:495-500

34. Tokunaga $M$, Egi $H$, Hattori $M$, Yoshimitsu $M$, Sumitani $D$, Kawahara T, et al. Approaching time is important for assessment of endoscopic surgical skills. Minim Invasive Ther Allied Technol. 2012;21:142-9.

35. Egi H, Okaiima $M$, Yoshimitsu $M$, Ikeda $S$, Miyata $Y$, Masugami H, et al. Objective assessment of endoscopic surgical skills by analyzing direction-dependent dexterity using the Hiroshima University Endoscopic Surgical Assessment Device (HUESAD). Surg Today. 2008;38:705-10.

36. Francis NK, Hanna GB, Cuschieri A. The Performance of Master Surgeons on the Advanced Dundee Endoscopic Psychomotor Tester. Arch Surg. 2002;137:841-4

37. Sánchez-Margallo JA, Sánchez-Margallo FM, Pagador JB, Bustos P, Moreno J. A laparoscopic hybrid simulator for skills assessment and augmented reality surgical applications. Int J Comput Assist Radiol Surg. 2011;6:S263-4.

38. Gunther S, Rosen J, Hannaford B, Sinanan M. The red DRAGON: a multi-modality system for simulation and training in minimally invasive surgery. Stud Health Technol Inform. 2007;125:149-54.

39. Pagador JB, Sánchez-Margallo FM, Sánchez-Peralta LF, Sánchez-Margallo JA, Moyano-Cuevas JL, Enciso S, et al. Decomposition and analysis of laparoscopic suturing task using tool-motion analysis (TMA): improving the objective assessment. Int J Comput Assist Radiol Surg. 2012;7: 305-13.

40. Brydges R, Classen R, Larmer J, Xeroulis G, Dubrowski A. Computer-assisted assessment of one-handed knot tying skills performed within various contexts: a construct validity study. Am J Surg. 2006;192:109-13.

41. Lievin M, Keeve E. Stereoscopic Augmented Reality System for Computer Assisted Surgery. Proceedings of the Computer Assisted Radiology and Surgery Congress, Berlin, Germany, 2001.

42. Estebanez B, Jimenez G, Muoz VF, Garcia-Morales I, Bauzano E, Molina J. Minimally invasive surgery maneuver recognition based on surgeon model. Proceedings of the International Conference on Intelligent Robots and Systems, St. Louis; MO, 2009.

43. Hwang $\mathrm{H}$, Lim J, Kinnaird C, Nagy AG, Panton ON, Hodgson AJ, et al. Correlating motor performance with surgical error in laparoscopic cholecystectomy. Surg Endosc. 2006;20:651-5.

44. Maier-Hein L, Franz A, Meinzer HP, Wolf I. Comparative assessment of optical tracking systems for soft tissue navigation with fiducial needles. Proceedings of SPIE, San Jose; CA, USA, 2008.

45. Ren H, Rank D, Merdes $M$, Stallkamp J, Kazanzides P. Development of a wireless hybrid navigation system for laparoscopic surgery. Stud Health Technol Inform. 2011;163:479-85.

46. Allen B, Nistor V, Dutson E, Carman G, Lewis C, Faloutsos P. Support vector machines improve the accuracy of evaluation for the performance of laparoscopic training tasks. Surg Endosc. 2010;24:170-8.

47. Groeger $\mathrm{M}$, Arbter $\mathrm{K}$, Hirzinger G. Motion Tracking for Minimally Invasive Robotic Surgery. In Bozovic V, editor. Medical Robotics. InTech, 2008.

48. Bouarfa L, Akman O, Schneider A, Jonker PP, Dankelman J. In-vivo real-time tracking of surgical instruments in endoscopic video. Minim Invasive Ther Allied Technol. 2012;21:129-34.

49. Tonet $\mathrm{O}$, Thoranaghatte RU, Megali G, Dario P. Tracking endoscopic instruments without a localizer: a shape-analysisbased approach. Comput Aided Surg. 2006;12:35-42.

50. Zhang XL, Payandeh S. Application of visual tracking for robot-assisted laparoscopic surgery. J Robot Syst. 2002;19: 315-28.

51. Doignon C, Nageotte F, Maurin B, Krupa A. Pose estimation and feature tracking for robot assisted surgery with medical imaging. In Kragic D, Kyrki V, editors. Unifying Perspectives in Computational and Robot Vision. Springer, 2007.

52. Voros S, Long JA, Cinquin P. Automatic detection of instruments in laparoscopic images: A first step towards high-level command of robotic endoscopic holders. Int J Rob Res. 2007;26:1173-90.

53. Speidel S, Delles M, Gutt C, Dillmann R. Tracking of Instruments in Minimally Invasive Surgery for Surgical Skill Analysis. Lect Notes Comput Sci. 2006;4091:148-55.

54. Sánchez-Margallo JA, Sánchez-Margallo FM, Pagador JB, Gómez EJ, Sánchez-González P, Usón J, et al. Video-based assistance system for training in minimally invasive surgery. Minim Invasive Ther Allied Technol. 2011;20:197-205.

55. Climent J, Hexsel RA. Particle filtering in the Hough space for instrument tracking. Comput Biol Med. 2012;42:614-23.

56. Oropesa I, Lamata P, Sánchez-González P, Pagador JB, García ME, Sánchez-Margallo FM, et al. Virtual realitysimulators for objective evaluation on laparoscopic surgery: 
Current trends and benefits. In Kim J, Editor Virtual reality. Intech Open Access Publisher, 2011.

57. Botden SM, Buzink SN, Schijven MP, Jakimowicz J. Augmented versus virtual reality laparoscopic simulation: what is the difference? A comparison of the ProMIS augmented reality laparoscopic simulator versus LapSim virtual reality laparoscopic simulator. World J Surg. 2007;31: 764-72.
58. Cristancho SM, Hodgson AJ, Panton ON, Meneghetti A, Warnock $G$, Qayumi $K$. Intraoperative monitoring of laparoscopic skill development based on quantitative measures. Surg Endosc. 2009;23:2181-90.

59. Dankelman J, Chmarra MK, Verdaasdonk EG, Stassen LP, Grimbergen CA. Fundamental aspects of learning minimally invasive surgical skills. Minim Invasive Ther Allied Technol. $2005 ; 14: 247-56$. 